\title{
Refractive index sensing of aqueous media based on plasmonic resonance in tapered optical fibres operating in the $1.5 \mu \mathrm{m}$ region
}

\author{
Natalia Díaz-Herrera ${ }^{\mathrm{a}, *}$, Agustín González-Cano ${ }^{\mathrm{a}}$, Diana Viegas ${ }^{\mathrm{b}, \mathrm{c}}$, José Luís Santos ${ }^{\mathrm{b}, \mathrm{c}}$, \\ María-Cruz Navarrete ${ }^{\mathrm{d}}$ \\ a Department of Optics, Escuela Universitaria de Óptica, Universidad Complutense, Arcos de Jalón 118, 28037 Madrid, Spain \\ b INESC Porto, Rua do Campo Alegre 687, 4169-007 Porto, Portugal \\ ${ }^{\mathrm{c}}$ Department of Physics, FCUP, Rua do Campo Alegre 687, 4169-007 Porto, Portugal \\ d Department of Optics, Facultad de CC Físicas, Universidad Complutense, Ciudad Universitaria s/n, 28040 Madrid, Spain
}

\section{A R T I C L E I N F O}

\section{Article history:}

Received 28 October 2009

Received in revised form 3 February 2010

Accepted 10 February 2010

Available online 19 February 2010

\section{Keywords:}

Surface plasmon resonance

Fibre optic sensors

Third telecommunications window

\begin{abstract}
A B S T R A C T
Experimental results are shown demonstrating that multiple surface plasma waves can be excited in optical fibres at the $1.5 \mu \mathrm{m}$ optical communications region for the range of refractive indices of aqueous media using doubly deposited tapered fibre structures, well known to exhibit small or zero sensitivity to polarization. Well-defined plasmon dips were obtained with high sensitivity to the surrounding refractive index. This characteristic, together with the substantial flexibility for local and distributed measurement associated with optical fibre sensing supported by the C-Band technology, indicate that these devices can be very advantageously used for chemical, biological and environmental sensing.
\end{abstract}

(C) 2010 Elsevier B.V. All rights reserved.

\section{Introduction}

Surface plasmon resonance (SPR) is a powerful concept for refractive index sensing, enabling measurement performances impaired by any other type of optical refractometer. The huge number of references in the literature proves that the phenomenon of the excitation of surface plasma waves in metallic layers can provide versatile and accurate measurements of a wide variety of physical, chemical and biological parameters [1-4].

Several different configurations have been proposed to excite plasmons, all of them based in evanescent coupling. The most popular in the biosensors community is based in attenuated total reflection in prisms (the so-called Kretchmann arrangement): it uses very often angular interrogation (although spectral interrogation is also possible), and in general requires bulky elements and more complicate setups. Optical fibres, on the other hand, usually employ spectral interrogation, and offer substantial advantages over the conventional configurations, such as all-fibre optical system, possibility of remote measurement and of operation in harsh environments, as well as the feasibility of sensor multiplexing. Polished or etched fibres have been commonly chosen as the basis of SPR sensors, almost always with one metallic layer deposited on them. The gold has been the most usual metal employed, however

\footnotetext{
* Corresponding author. Tel.: +34 91394 5010; fax: +34 913944683 .

E-mail address: ndiazher@fis.ucm.es (N. Díaz-Herrera).
}

there are others metals that can be use to excite plasmons. Many sensors based on this technology have been presented in the literature in the last years, and some comprehensive reviews have also appeared to give account of the many advances of this progressive research field.

In recent years, uniform-waist tapered fibres have been proposed to develop more compact and efficient SPR sensors, and it has been shown that this kind of elements can be very advantageously used in chemical and biological sensing [5]. The authors introduced the idea of adding a second deposited dielectric layer on uniform-waist tapers, and experimentally demonstrate that this second layer permits a better tuning of the resonance wavelength to the range of refractive indices of interest. Different geometrical dispositions of the deposits has also been investigated and the characterization of the response of the devices was made, especially in terms of number of plasmons excited, polarization dependence and sensitivity when operating as refractometers. These devices have already been detailedly depicted in different references of the literature [6-9].

The SPR sensors for biological applications have been focused to detect refractive indices of aqueous media, which means that they are sensitive in the range of the refractive index of water $(\approx 1.33$ at visible wavelengths). However, in the vast majority of the studies reported the optical fibre based SPR sensors operate around $800 \mathrm{~nm}$, which is a wavelength region where the availability of high-performance fibre optic components is reduced. To take advantage of the highly developed fibre optic technology, SPR fibre 
sensing must migrate to the C-band of optical communications, around $1500 \mathrm{~nm}$, surprisingly a move that has been quite slow so far. Very few devices have been proposed to achieve that goal, and in general they are more complicate and less versatile that the one we present. With bulk optics, SPR films have been designed to exhibit resonances in the $1500 \mathrm{~nm}$ region [10], and in this wavelength window a fibre optic taper-based SPR sensing head was reported for refractive index detection in the region of 1.44 [11]. Recently, it was demonstrated a $1500 \mathrm{~nm}$ fibre optic SPR sensor for aqueous media refractive index measurement based on a combination of a tilted fibre Bragg grating and a SPR film structure deposited at a lapped cladding region of the fibre [12]. The results reported indicate the feasibility of this approach, but it would be interesting to have simpler sensing devices and less sensitive to the polarization properties of the light.

In that sense, in this work we theoretically and experimentally study SPR sensors based on doubly deposited uniform-waist tapered fibres operating around $1500 \mathrm{~nm}$ and sensitive to the refractive index of aqueous media, and experimental evidence of multiple plasmon resonance for those values is presented, showing in this way how these very compact and versatile devices can be the basis of a new generation of chemical and biological sensors.

\section{Configuration and fabrication of sensors}

Doubly deposited uniform-waist tapered optical fibres (DLUWTs) have shown their good performance and their possibilities in the past [6-9]. However, the range of operating wavelengths for the detection of refractive indices similar to that of water has always been the very near infrared, around $800 \mathrm{~nm}$. DLUWTs are versatile elements, and the selection of the parameters of the layers in the designing stage has been made to tune the plasmon resonances for the desired ranges of wavelengths and refractive indices.

To move the response of the devices to the C-Band of optical communications we again take advantage of that versatility. In the first place we select the thickness and the refractive indices of the materials to be deposited (one metal plus one dielectric). We have used the existing theoretical models developed by us to do that [13]. These are very simple models, quasi-geometrical, that employ flat layers as an approximation, but they are very convenient to determine the position of the main plasmon resonance in terms of the configuration of the DLUWT. The goal of the use of those models is to select the thickness and the materials of the layers to be deposited on the taper, thus achieving that the resonance wavelength appears on the desired region for the desired values of the refractive index of the surrounding medium. Finer details of the response of the system (like multiple resonances when asymmetric layers are used) are not to be predicted by the model, but this is not a very important point in the design stage. Also, the model can be complicated if we desire this kind of accurate prediction.

We tried with Gold, which is the most usual material for SPR, but also with Aluminum, which has been our most used metal for SPR device fabrication with very good performances. For the dielectric we selected Titanium Dioxide, again taking advantage of our previous expertise on its processing.

We show in Fig. 1 the results of the simulation of the behavior of sensors based on the deposition of only one layer of Gold. We have taken a typical taper waist value of $35 \mu \mathrm{m}$ and that of water as outer refractive index. A very remarkable result is that we cannot possibly obtain resonances around $1.5 \mu \mathrm{m}$ if we only use a single layer of Gold, which is commonest in SPR sensors. As it is shown, as the thickness of the gold layer is increased, the position of the minimum associated to the plasmon is displaced towards higher wavelengths but the depth of this minimum quickly decreases until it is barely noticeable and inoperative.

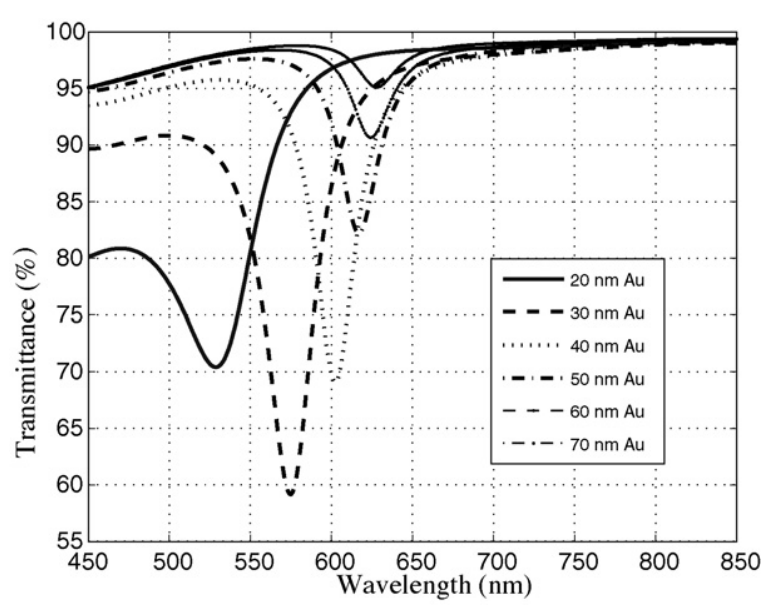

Fig. 1. Simulation of the behavior of transducers with a single layer of Au.

Then, the second layer of our developed configuration is, therefore, crucial to obtain plasmons around the water refractive index for the range of $1.5 \mu \mathrm{m}$, and DLUWTs are in this way shown to be the best option to achieve the goal. In Fig. 2 the results of the simulations for double depositions appear. It can be seen that, by adequately selecting the thickness of the layers, it is possible to tune the position of the resonance to the desired wavelength region, when the outer refractive index considered is that of aqueous media. In addition, with a second layer it is possible to enhance the depth of the minimum to very low transmittance values. Also, when using Gold, as it is well-known, it is necessary to add a very thin layer of chromium to improve adherence to the silica of fibres. This is not necessary with aluminum. Additionally, the combination of $\mathrm{Al} / \mathrm{TiO}_{2}$ has been used in other contexts for years and it has proved to be very convenient in practical terms. All these reasons prove that $\mathrm{Al} / \mathrm{TiO}_{2}$ DLUWTs are the best choice, although $\mathrm{Au} / \mathrm{TiO}_{2}$ can also be tested.

To produce the operating devices, we have worked with a travelling-burner scheme to fabricate uniform-waist, almost adiabatic, tapers. This procedure has become standard and details of it can be found elsewhere $[6,14]$. A standard telecommunications optical fibre (SMF-28) was used. Two different deposits were fabricated by sputtering to excite plasmon resonances at wavelengths

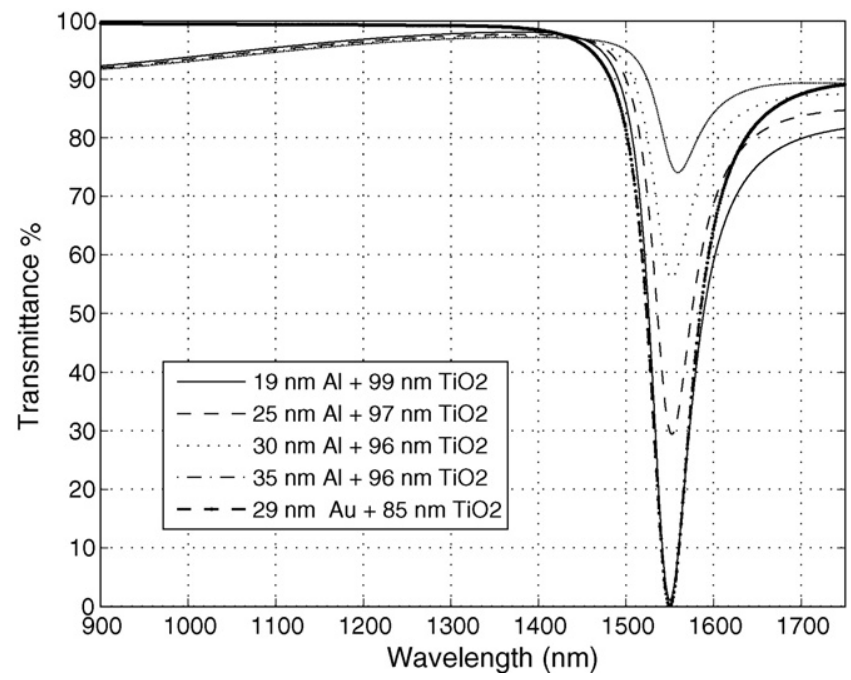

Fig. 2. Simulation of the behavior of doubly deposited uniform-waist tapered layers with thicknesses calculated to produce excitation of plasmons in the region of interest. 


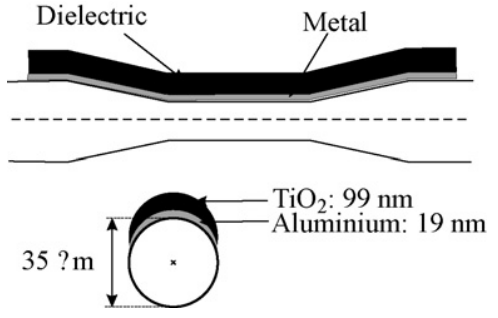

Fig. 3. Scheme of the configuration of one of the sensors fabricated and characterized.

around $1550 \mathrm{~nm}$ : a triple deposit with $\mathrm{Cr}(2 \mathrm{~nm})$ plus $\mathrm{Au}(26 \mathrm{~nm})$ plus $\mathrm{TiO}_{2}(92 \mathrm{~nm})$ and a double deposit with $\mathrm{Al}(19 \mathrm{~nm})$ plus $\mathrm{TiO}_{2}$ $(99 \mathrm{~nm})$. As expected, this second set of layers has proved to be the most efficient. We show in Fig. 3 the scheme of one of the DLUWTs employed, with the depicted thicknesses and materials.

We have chosen to use asymmetrically deposited sensors. In this case, multiple resonances appear, due to the non-constancy of the thickness of the layers, and the dependence on the polarization of the incident light still exists, but the responses of the sensors are still satisfactory even if we do not control polarization at all $[7,8]$. We can have, however, fully symmetric deposits that show no polarization dependence at all and a single plasmon resonance [9], thus greatly simplifying the setup. We have, therefore, included, in the characterization setup, depicted in the next paragraph, some polarization-controlling elements to improve the measurements that can be suppressed in real applications.

\section{Characterization of the sensors and experimental evidence of plasmon resonance}

The response of the sensors to the variation of the outer refractive index was characterized in the laboratory in order to determine the appearance of plasmon resonances and the displacements of the SPR dips when the refractive index of the external medium varies. The light source was a Tungsten lamp, directly connected to the sensor. As it was said before, polarization-controlling elements (a polarizer and Lefebvre loops) were included. At the output, the transducer was connected to an Avantes spectrometer (AvaSpec 2048-2) to register the transmittance spectra. The refractive index of the external medium was changed from 1.3320 up to 1.3375 , by controlled addition of ethylene glycol to water. The value of the refractive index of the mixture was measured by an Abbe refractometer. These are values for the wavelengths typical of visible region. It is obvious that we expect some change when moving to $1.5 \mu \mathrm{m}$, but we have decided to keep as a reference these directly measured values. If we take into account both experimental measurements and semi-empirical laws in the literature $[15,16]$, we see that the value for the refractive index of water around $1.5 \mu \mathrm{m}$ is about 1.318 . The indices for the corresponding mixtures with ethylene glycol will vary in a much similar way to the variation for the visible region when the concentrations are changed. The radiation travelling in the fibre is going to "see" refractive indices about 1.32 that correspond to the refractive indices about 1.34 in the visible region, but the major point is that well-defined plasmons for both combinations of layers were obtained, in that range of interest. In Fig. 4 the transmittance curves for the $\mathrm{Al} / \mathrm{TiO}_{2}$ sensor are shown. Multiple plasmon dips can be observed. They are displaced when the refractive index of the external medium varies.

In Fig. 5 it is shown the displacement of the wavelength associated to the main plasmon resonance (that of the absolute minimum in the transmittance) as the refractive index of the surrounding medium changes. As it can be seen, linearity is good, and sensitivity is substantially higher than that typically achieved with the

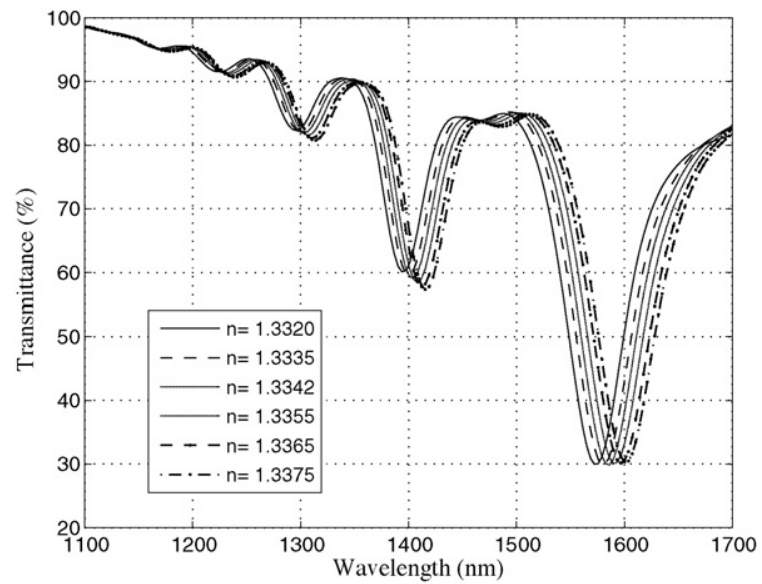

Fig. 4. Experimental curves showing multiple plasmon resonances and their displacement as the refractive index of the outer medium varies. The values of the refractive indices given are those measured with the Abbe refractometer.

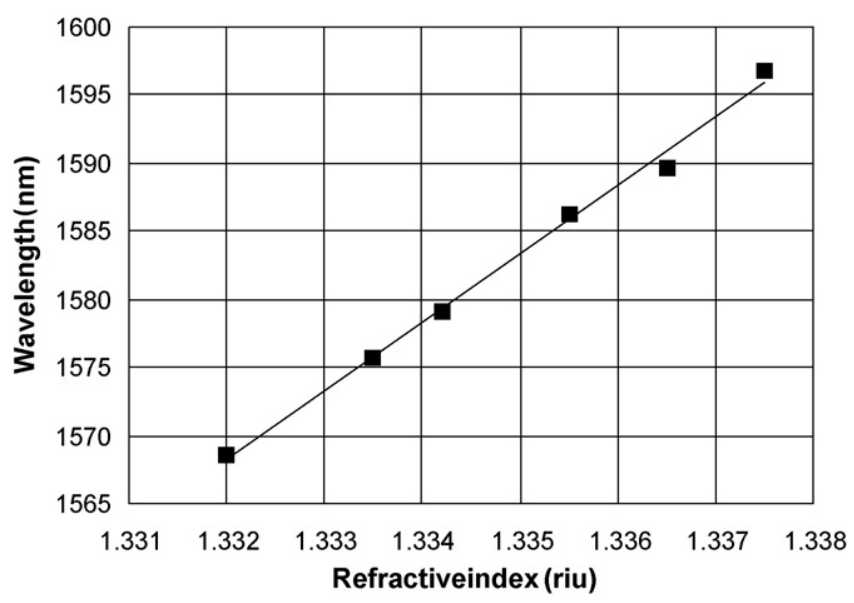

Fig. 5. Dependence on the position of the wavelength associated to the main plasmon resonance with outer refractive index. The values of the refractive indices given on the $\mathrm{x}$-axis are those measured with the Abbe refractometer.

same elements in the $800 \mathrm{~nm}$ region ( $\sim 5000 \mathrm{~nm} /$ riu at $1500 \mathrm{~nm}$ vs. $\sim 3000 \mathrm{~nm} /$ riu at $800 \mathrm{~nm}$ ). These estimations are made taking as reference the values of the indices in the visible region. The sensitivity in terms to the values corresponding to each of the wavelengths in the NIR region is expected to be very much the same. In any case, the response is better when radiation of $1.5 \mu \mathrm{m}$ is used to interrogate the plasmons.

\section{Conclusions}

The experimental results obtained in this work confirm the feasibility of the proposed approach to achieve the goal of operative SPR sensors for aqueous medium working in the wavelength range of the third communication window, with plasmon resonances around $1.5 \mu \mathrm{m}$ for refractive indices of the order of 1.33 . These results open a wide range of possibilities of use of these elements as SPR transducers in the very exigent field of chemical and biological sensing.

The very good performance of DLUWTs, the versatility of their design, the simplicity of their production, the small or zero dependence on polarization and the subsequent elimination of all polarization-controlling elements and the availability of highperformance fibre optic components for operation at the third 
communication window, indicate that compact and cost effective SPR sensors can be developed using this approach.

Also, the application to these sensing structures of interrogation techniques widely developed in the context of optical fibre sensing shall permit the development of high-performance SPRbased sensing systems for remote and multi-point measurement of chemical and biochemical parameters.

\section{Acknowledgments}

This work has been partially supported by Spanish Government research project NESTOR, ref. CTM2004-03899; Comunidad de Madrid research project FUTURSEN, ref. S-0505/AMB-0374 and by Proyecto de Investigación Santander/Complutense, ref. PR34/0715886. This work was supported partially by the Portuguese Government - Fundação para a Ciência e Tecnologia (FCT) through the grant SFRH/BD/30086/2006. N. Díaz-Herrera is thankful for the grant within the program 'Becas Internacionales Universidad Complutense/Empresa Flores Valles 2008'. The authors wish to thank Carmen Cosculluela from Departamento de Física Aplicada of the Universidad de Zaragoza (Spain) and Javier de la Cruz from INESC Porto (Portugal) for helping us with the devices elaboration.

\section{References}

[1] R.C. Jorgenson, S.S. Yee, A fiber-optic chemical sensor based on surface plasmon resonance, Sensors and Actuators B 12 (1993) 213-220.

[2] J. Homola, Surface plasmon resonance sensors for detection of chemical and biological species, Chemical Review 108 (2008) 462-493.

[3] A.K. Sharma, R. Jha, B.D. Gupta, Fiber-optic sensors based on surface plasmon resonance: a comprehensive review, IEEE Sensors Journal 7 (2008) 1118-1128.

[4] A. Leung, P.M. Shankar, R. Mutharasan, A review of fiber-optic biosensors, Sensors and Actuators B 125 (2007) 688-703.

[5] D. Monzón-Hernández, J. Villatoro, High-resolution refractive index sensing by means of a multiple peak surface plasmon resonance optical fiber sensor, Sensors and Actuators B 115 (2006) 227-231.

[6] F.J. Bueno, Ó. Esteban, N. Díaz-Herrera, M.C. Navarrete, A. González-Cano, Sensing properties of asymmetric double-layer covered tapered fibres, Applied Optics 43 (2004) 1615-1620.

[7] A. González-Cano, F.J. Bueno, Ó. Esteban, N. Díaz-Herrera, M.C. Navarrete, Multiple surface-plasmon resonance in uniform-waist tapered optical fibers with an asymmetric double-layer deposition, Applied Optics 44 (2005) 519-526.

[8] Ó. Esteban, N. Díaz-Herrera, M.C. Navarrete, A. González-Cano, SPR sensors based on uniform-waist tapered fibers in reflective configuration, Applied Optics 45 (2006) 7294-7298.

[9] M.C. Navarrete, N. Díaz-Herrera, A. González-Cano, Ó. Esteban, “A polarizationindependent SPR fiber sensor", Plasmonics, doi:101007/s11468-009r-r9108-0.

[10] S. Pastkovsky, A.V. Kabashin, M. Meunier, J.H.T. Luong, Near-infrared surface plasmon resonance sensing on a silicon platform, Sensors and Actuators B 97 (2004) 409-414.

[11] A. Díez, M.V. Andrés, J.L. Cruz, In-line fiber-optic sensors based on the excitation of surface plasma modes in metal-coated tapered fibers, Sensors and Actuators B 73 (2001) 95-99.
[12] T. Allsop, R. Neal, S. Rehman, D.J. Webb, D. Mapps, I. Bennion, Generation of infrared surface plasmon resonances with high refractive index sensitivity utilizing tilted fiber Bragg gratings, Applied Optics 46 (2007) 5456-5460.

[13] Ó. Esteban, M.C. Navarrete, A. González-Cano, E. Bernabéu, Simple model of compound waveguide structures used as fiber-optic sensors, Optics and Lasers in Engineering 33 (2000) 219-230.

[14] J. Villatoro, D. Monzón-Hernández, E. Mejía, Fabrication and modelling of uniform-waist single-mode tapered optical fiber sensors, Applied Optics 42 (2003) 2278-2283.

[15] V. Melchor Centeno, The refractive index of liquid water in the Near Infra-Red Spectrum, Journal of the Optical Society of America 31 (1941) 244-247.

[16] L. Thormählen, J. Straub, U. Grigull, Refractive index of water and its dependence on wavelength, temperature and density, Journal of Physical and Chemical Reference Data 14 (1985) 933-945.

\section{Biographies}

Natalia Díaz-Herrera received the MSc degree in physics from the Universidad Autónoma de Madrid, Madrid, Spain, in 2001 and the PhD degree in applied optics from the Universidad Complutense de Madrid (UCM) in 2005. In 2006 she joined the Optics Department of the UCM as a lecturer, where she has carried out her research and teaching activities. She has been involved in several European and Spanish projects. Her research interests are optical fibre sensors, specifically for environmental applications.

Agustín González-Cano was born in Madrid (Spain) in 1964. He received his MSc in Physics (1987) and PhD in Advanced Optics (1994) in the Universidad Complutense de Madrid (UCM), where he has developed his research and teaching activities during the last 22 years. Currently he is a professor in the School of Optics and Optometry of the UCM, where his main teaching subjects are Ophthalmic Optics, and Geometrical Optics. His research interests are basically optical sensors, and specifically fibre-optic sensors for environmental applications. In the past he has also been working in image processing, fringe pattern analysis and photoelasticity. In recent times he is also interested in research on the history of Optics. He has published more than 30 papers and has been involved in many different research projects, including some European Union-funded ones. He is a member of the Spanish Optics Association (SEDOPTICA)

Diana Catarino das Neves Viegas graduated in physics in 2003 and received the MSc degree in computational methods in science and engineering in 2006, both from the University of Porto, Porto, Portugal. Her main interests are optical physics, including interferometry. She is doing her PhD work on microfabrication of optical devices for sensing applications in Faculty of Sciences of University of Porto since 2007.

José Luis Santos graduated in Physics (Optics and Electronics) by University of Porto (1983). PhD from the same University (1993). His main research interests are related to optical fibre technology and optical fibre sensing. He holds the position of associate professor of Physics Department of University of Porto, and he is also manage of INESC Porto Optoelectronics and Electronic Systems Unit. He is member of OSA and SPIE.

María-Cruz Navarrete received her MSc degree in Physics in 1986, and her PhD degree of Physics in 1994 in the Universidad Complutense de Madrid (UCM, Spain). She joined Optics Department (UCM) in 1990 where she has carried out her research and teaching activities. She has been involved in fibre optic research and in severa European and Spanish projects. During the last 12 years she has been working in optical fibre sensors, mainly applied to environmental measurements. Nowadays she is an assistant professor in the Optics Department and a member of the Applied Optics Complutense research group. 